\title{
REVIEWS
}

Adv Clin Exp Med 2016, 25, 6, 1337-1344

(C) Copyright by Wroclaw Medical University

DOI: $10.17219 /$ acem/64942

ISSN 1899-5276

\author{
Andrzej Mital ${ }^{\mathrm{A}-\mathrm{F}}$
}

\section{Acquired von Willebrand Syndrome}

Department of Hematology and Transplantology, Medical University of Gdańsk, Poland

A - research concept and design; $\mathbf{B}$ - collection and/or assembly of data; $\mathbf{C}$ - data analysis and interpretation;

D - writing the article; $\mathbf{E}$ - critical revision of the article; $\mathbf{F}$ - final approval of article

\begin{abstract}
Acquired von Willebrand syndrome is a rare hemorrhagic diathesis, with clinical symptoms similar to those associated with the inherited form von Willebrand disease. This syndrome is characterized by a lack of previous bleeding symptoms, negative familial history, and occurrence in a relatively older age. Most commonly, acquired von Willebrand syndrome develops in the course of other conditions, such as lymphoproliferative, myeloproliferative, cardiovascular and autoimmune disorders; additionally, it can be associated with some non-hematological malignancies and use of certain prescription drugs. Pathogenesis of von Willebrand syndrome is complex and not fully understood. Deficiency or impaired activity of von Willebrand factor can result from the presence of specific antibodies against this factor, its adsorption onto the surfaces of neoplastic cells, mechanic injury or proteolysis. Diagnosis is based on the measurements of plasma concentration and the activity of von Willebrand factor and multimer analysis. Management of acquired von Willebrand syndrome includes the therapy of the underlying disease and the control or prevention of bleeding. Hemostatic drugs that are most commonly prescribed in this syndrome include desmopressin, von Willebrand factor concentrates, recombinant activated factor VII, intravenous immunoglobulin and adjunctive antifibrinolytic therapy. Additionally, plasmapheresis is required in some cases (Adv Clin Exp Med 2016, 25, 6, 1337-1344).
\end{abstract}

Key words: diagnosis, management, prevalence, acquired von Willebrand syndrome.

Acquired von Willebrand Syndrome (AvWS) is a rare bleeding disorder with the profile of clinical symptoms and laboratory abnormalities similar to that observed in the case of inherited von Willebrand disease (vWD). Characteristic features of AvWS include lack of previous clinical bleeding abnormalities, late onset and negative family history $[1,2]$. AvWS usually occurs concomitantly to other conditions.

\section{Epidemiology}

The disease was described for the first time in 1968 , in a 7-year-old boy with systemic lupus erythematosus [3]. In 2000, the international registry run by the International Society of Thrombosis and Hemostasis (ISTH) included 186 patients with AvWS, and it is estimated that a total number of individuals with this condition exceeds $300[1,4]$. Based on the data from the ISTH reg- istry, AvWS seems to occur most often in patients with lymphoproliferative (48\%) and myeloproliferative neoplasms (15\%), cardiovascular diseases (21\%), solid tumors (5\%) and autoimmune disorders (2\%) [4-6].

The most common lymphoproliferative disorders than can be complicated by AvWS are monoclonal gammapathy of undetermined significance (MUGS) and multiple myeloma. Essential thrombocythemia is a myeloproliferative neoplasm, which co-exists with this condition most frequently $[5,6]$. AvWS observed during the course of hypothyroidism, uremia, after the administration of some agents or spontaneously, without other comorbidities, is of markedly rarer evidence $[4,7,8]$. A full list of diseases that have been shown to co-exist with AvWS is presented in Table 1.

Owing to the recent increase in the incidence of AvWS in cardiovascular patients, the abovementioned list is likely to change $[9,10]$. AvWS may emerge at any age; the mean age at diagnosis 
Table 1. Disorders associated with AvWS $[4-6,14]$

\begin{tabular}{|c|c|}
\hline Primary underlying conditions & Examples \\
\hline Lymphoproliferative disorders & $\begin{array}{l}\text { multiple myeloma } \\
\text { monoclonal gammapathy of undetermined significance (mgus) } \\
\text { Waldenström macroglobulinemia } \\
\text { chronic lymphocytic leukemia } \\
\text { hairy cell leukemia } \\
\text { non Hodgkin lymphomas } \\
\text { acute lymphoblastic leukemia }\end{array}$ \\
\hline Myeloproliferative disorders & $\begin{array}{l}\text { essential thrombocythemia } \\
\text { polycythemia vera } \\
\text { spontaneous myelofibrosis } \\
\text { chronic myeloid leukemia }\end{array}$ \\
\hline Non-hematological malignancies & $\begin{array}{l}\text { Wilms' tumors } \\
\text { cancers/solid tumors } \\
\text { primitive neuroectodermal tumors }\end{array}$ \\
\hline Autoimmune/endocrine disorders & $\begin{array}{l}\text { systemic lupus erythematosus } \\
\text { other connective tissue disorders } \\
\text { graft versus host disease } \\
\text { hypothyroidism }\end{array}$ \\
\hline Cardiovascular diseases (inherited, acquired) & $\begin{array}{l}\text { ventricular septal defects } \\
\text { atrial septal defects } \\
\text { aortic stenosis } \\
\text { mitral valve prolapse } \\
\text { endocarditis } \\
\text { angiodysplasia } \\
\text { systemic atherosclerosis }\end{array}$ \\
\hline $\begin{array}{l}\text { Drugs and other therapeutic agents: } \\
\text { antibiotics } \\
\text { antiepileptic agents } \\
\text { plasma volume expanders } \\
\text { coagulation factors }\end{array}$ & $\begin{array}{l}\text { ciprofloxacin, griseofulvin, tetracycline } \\
\text { valproic acid } \\
\text { hydroxyethyl starch (hes) } \\
\text { recombinant factor viii }\end{array}$ \\
\hline Other & $\begin{array}{l}\text { uremia } \\
\text { infections (viral, parasitic) } \\
\text { diabetes mellitus } \\
\text { hemoglobinopathies } \\
\text { sarcoidosis } \\
\text { ehlers-danlos syndrome } \\
\text { telangiectasia } \\
\text { ulcerative colitis } \\
\text { liver cirrhosis } \\
\text { turner syndrome } \\
\text { lactoferrin deficiency } \\
\text { myelodysplastic syndrome }\end{array}$ \\
\hline
\end{tabular}

is 62 years (range 2-96 years) [2]. The true prevalence of AvWS is difficult to establish due to its low detection rate and frequent misdiagnoses.

\section{Pathogenesis}

The pathogenesis of AvWS is complex and not completely understood. In most cases, the synthe- sis of von Willebrand factor (vWF) in megakaryocytes and endothelial cells and its release to circulation are normal [11]. The only exception pertains to patients with hypothyroidism in whom these processes are disrupted, which results in a decrease in vWF (vWF:Ag) concentration, its lower activity (vWF:RCo) and resistance to desmopressin (DDAVP) $[2,12,13]$. Decreased concentration of $\mathrm{vWF}$ in patients in whom the synthesis and re- 
Table 2. Pathogenic mechanisms of AvWS and underlying disorders [14]

\begin{tabular}{|l|l|}
\hline Mechanism & Underlying disorder \\
\hline Presence of autoantibodies & $\begin{array}{l}\text { lymphoproliferative disorders } \\
\text { non-hematological malignancies } \\
\text { autoimmune diseases }\end{array}$ \\
\hline Adsorption on cancer cells or other cells & $\begin{array}{l}\text { lymphoproliferative disorders } \\
\text { myeloproliferative disorders } \\
\text { non-hematological malignancies } \\
\text { cardiovascular diseases (high shear stress) } \\
\text { therapeutic agents: hes }\end{array}$ \\
\hline $\begin{array}{l}\text { Loss of high-molecular--weight multimers } \\
\text { (damage/proteolysis) }\end{array}$ & $\begin{array}{l}\text { myeloproliferative disorders } \\
\text { excessive shear stress: } \\
\text { cardiovascular diseases (cardiac defects, endocarditis) } \\
\text { vascular malformations (osler's disease, kasabach-merritt syndrome) } \\
\text { atherosclerosis } \\
\text { hemoglobinopathies } \\
\text { uremia } \\
\text { therapeutic agents: ciprofloxacin }\end{array}$ \\
\hline Decreased synthesis of vWF & hypothyroidism \\
\hline
\end{tabular}

lease of this factor are normal can be explained by one of the following mechanisms $[1,14,15]: 1)$ development of specific or non-specific autoantibodies that impair the function of vWF and enhance its clearance, 2) adsorption of vWF on cancer cells, also resulting in its enhanced clearance, and 3) loss of high-molecular-weight (HMW) multimers of vWF (HMW vWF), resulting from their exposure to high shear stress or proteolysis. The exact pathogenic mechanism of AvWS is often specific for a primary condition in the course of which it has developed (Table 2).

In the first mechanism, normally synthesized vWF is promptly eliminated from circulation due to the presence of specific or non-specific autoantibodies. While most autoantibodies against vWF are IgG, antibodies from other classes have also been implicated sporadically [16]. Anti-vWF autoantibodies can interact with active domains of the factor responsible for binding to collagen and platelets, without interfering with factor VIII [17-19]. Upon binding to functional or non-functional domain of vWF, autoantibodies form immunological complexes which are promptly removed from circulation via the reticular endothelial system. As a result, both the activity and concentration of vWF (vWF:RCo and vWF:Ag) are decreased, whereas the level of vWF propeptide (vWFpp, also referred to as vWF:AgII) is normal or even elevated [20].

The second mechanism, associated with the adsorption of vWF on cancer cells and its resultant enhanced clearance, is typically observed during the course of lymphoproliferative neoplasms (mul- tiple myeloma, Waldenström macroglobulinemia, malignant lymphoma, hairy cell leukemia) and adrenal cancer. Adsorption of vWF on cancer cells can be confirmed immunologically (immunofluorescence with anti-vWF antibodies) and by means of flow cytometry $[21,22]$. The same mechanism has also been involved in the case of myeloproliferative neoplasms. Adsorption of HMW vWF in activated platelets of patients with essential thrombocythemia results in thrombocytopenia, and the platelet count was shown to correlate inversely with plasma concentration of vWF multimers [23]. Adsorption of HMW vWF has also been reported in many other conditions associated with platelet activation. One example is aortic valve stenosis, in the course of which high shear stress causes activation of thrombocytes with resultant adsorption of HMW vWF on their surface [24]. Furthermore, vWF was also shown to be adsorbed on macromolecules of hydroxyethyl starch (HES) [25].

The third mechanism is associated with either mechanical or proteolytic loss of HMW vWF. In patients with aortic valve stenosis, vWF multimers can be subject to mechanical damage or proteolysis with ADAMTS-13 metalloproteinase [26-28]. Proteolysis of HMW vWF during the course of essential thrombocythemia may be triggered by platelet-released calcium ions, causing the activation of proteases and elastases. In such cases, proteolysis can be confirmed based on the presence of enzymatically-digested fragments of HMW vWF [29]. Excessive pathological proteolysis of HMW vWF may also occur in the course of uremia, pancreatitis, liver cirrhosis, leukemia and after the adminis- 
tration of some therapeutic agents, e.g. ciprofloxacin [30-32].

Patients in whom AvWS results primarily from adsorption of HMW vWF or loss thereof, typically present with lower activity of vWF (vWF:RCo) and its relatively normal concentration (vWF:Ag) [1].

Aside from the abovementioned ones, also other, still not completely understood, pathomechanisms of AvWS exist. For example, in patients with Wilms' tumors, who neither synthesize anti-vWF autoantibodies nor show the evidence of vWF adsorption, decreased activity of this factor is postulated to be linked to exposure to hyaluronic acid synthesized in large amounts by the nephroblastoma cells $[33,34]$.

Finally, it should be remembered that pathogenesis of AvWS associated with many primary conditions may be complex, involving more than one of the mechanisms mentioned above.

\section{Diagnosis}

Initial evaluation of patients with suspected AvWS is similar as in the case of individuals with inherited vWD. Important factors differentiating AvWS from vWD are: negative family history, lack of previous bleeding disorders and late onset of clinical symptoms. Usually, the results of basic laboratory tests are insufficient to distinguish between inherited vWD and AvWS. Diagnostic protocol should include history taking and examination for primary conditions that typically co-exist with AvWS. All patients with suspected vWD and negative family history of bleeding disorders should be screened for most common comorbidities predisposing AvWS [35]. Basic tests used in the diagnostics of hemostatic disorders and primary diseases predisposing to AvWS are listed in Table 3.
Bleeding time and occlusion time (PFA-100) are usually prolonged in AvWS, which points to the presence of a primary hemostatic disorder [36]. The concentration of vWF (vWF: Ag) and procoagulant activity of factor VIII is typically normal or slightly decreased. However, the activity of $\mathrm{vWF}$ as ristocetin cofactor (vWF:RCo) and its ability to bind to collagen (vWF: CB) are evidently decreased [37]. Also, vWF:RCo/vWF:Ag and vWF:RCo/vWF:CB ratios should be determined, as these parameters are typically decreased during the course of AvWS [38]. In a retrospective study conducted by Tiede et al. [39], all patients with cardiovascular diseases and most individuals with lymphoproliferative neoplasms presented with normal or elevated values of vWF:Ag, vWF:RCo and vWF:CB, and frequently showed abnormal vWF:RCo/vWF:CB and vWF:RCo/vWF:Ag ratios. These findings confirm the usefulness of collagen binding assay, which should be routinely used in the evaluation of patients with suspected AvWS.

An important test helpful in distinguishing between vWD and AvWS is vWF multimer analysis (electrophoresis and markedly less widespread densitometry) [40, 41]. Usually, AvWS is associated with complete loss of HMW vWF or decreased level thereof [19]. Owing that HMW vWF deficiency may be also observed in some patients with normal levels of vWF:RCo, vWF:Ag and vWF:CB, as well as in persons with normal vWF:RCo/ vWF:Ag and vWF:CB/vWF:Ag ratios, all subjects with suspected AvWS should be subjected to multimer analysis $[9,35,39]$. Although most patients with AvWS present with type 2 vWD [19], type 1 or type $3 \mathrm{vWD}$ can be also detected in individuals in whom AvWS is associated with abnormal synthesis of vWF (e.g. hypothyroidism) [19, 42].

Determination of vWFpp provides insight into $\mathrm{vWF}$ biosynthesis. If the production of $\mathrm{vWF}$ is

Table 3. Diagnostic tests used to detect AvWS and concomitant underlying disorders

\begin{tabular}{|l|l|}
\hline Hemostasis & Concomitant diseases \\
\hline Bleeding time/APTT & complete blood count with peripheral smear and platelet count \\
Occlusion time (PFA-100) & proteinogram \\
Activity of vWF (vWF:RCo) & immunofixation \\
Concentration of vWF (vWF:Ag) & tsh, triiodothyronine, thyroxin \\
Activity of factor VIII (FVIII:C) & autoimmune disorders: screening for antinuclear antibodies \\
Collagen binding assay (vWF:CB) & $\quad$ immunoblotting, ana-hep2, anti-native dna antibodies \\
Ristocetin-induced platelet aggregation & cardiovascular diseases: echocardiography, examination of vessels \\
vWF:RCo/vWF:Ag ratio & hematological malignancies: bone marrow biopsy, genetic/molecular tests \\
vWF:CB/vWF:Ag ratio & - jak2, bcr/abl mutations, bone marrow/peripheral blood phenotype, \\
Multimer analysis (plasma, platelets) & biochemical tests - ldh, beta-2 microglobulin, granulocyte alkaline \\
vWF propeptide (vWFpp) & phosphatase, imaging studies - ultrasonography, computed tomography, \\
Anti-vWF antibodies & histopathologic examination of lymph node or other tissue specimens) \\
Genetic tests: DNA sequencing & \\
\hline
\end{tabular}


impaired, concentration of vWFpp is also lower; this is observed in type 1 and $3 \mathrm{vWD}$, as well as in AvWS associated with hypothyroidism. In turn, enhanced synthesis of vWFpp and a relative increase in its concentration in relation to vWF:Ag co-exist with faster clearance of vWF observed in the course of AvWS as well as in some cases of type $1 \mathrm{vWD}$ and type $3 \mathrm{vWD}$ with synthesis of alloantibodies [43].

Since determining vWFpp/vWF:Ag ratio is not always sufficient to distinguish between inherited vWD and AvWS, routine use of this test is no longer recommended [35].

All patients with suspected AvWS should be tested for anti-vWF autoantibodies [1, 18]. Although autoantibodies play a role in the pathogenesis of AvWS in only some patients, especially those with concomitant lymphoproliferative neoplasms [35], their presence is associated with worse prognosis, namely with a tendency to cause severe bleeding $[18,39]$. Anti-vWF autoantibodies can be detected during a mixing study. During this test, the patient plasma is mixed with normal plasma, and vWF activity in the mixture is determined after a few hours of its incubation at $37^{\circ} \mathrm{C}$. In contrast to individuals with acquired $\mathrm{vWD}$, only a small proportion of patients with AvWS present with neutralizing antibodies. Presence of non-neutralizing antibodies that do not block the activity of vWF but enhance its clearance cannot be detected during a mixing study [44]. However, these antibodies can be determined with an enzyme-linked immunosorbent assay (ELISA) [11,39].

\section{Treatment}

Treatment of AvWS is oriented at: 1) control of active bleeding, 2) prevention of bleeding in high-risk patients, e.g. individuals requiring an invasive procedure, and 3) achieving remission of a primary condition occurring concomitantly to AvWS $[1,35]$.

Whenever the patient does not show the signs of active bleeding and the procedures with increased risk of bleeding can be postponed, treatment of a primary underlying condition is always a priority, since its remission is frequently associated with resolution of AvWS [45]. Individuals with autoimmune disorders are usually treated with glucocorticoids and other immunosuppressive agents (cyclophosphamide, azathioprine, cyclosporine) and patients with lymphoproliferative neoplasms (lymphoma, multiple myeloma) receive chemotherapy/immunochemotherapy, radiotherapy and immunomodulatory therapy. Efficient treatment of B-cell lymphoma and multiple myeloma was shown to result in complete remission of AvWS in $35-70 \%$ of the cases $[18,39]$. In subjects with MGUS, who usually do not require any specific treatment, therapy with intravenous immunoglobulins (in IgG-MGUS) or plasmapheresis (in IgM-MGUS) can be implemented whenever the signs of bleeding disorder emerged in the course of AvWS [35].

Patients with myeloproliferative neoplasms are typically treated with cytoreductive agents, tyrosine kinase inhibitors; also bone marrow allotransplantation can be considered whenever progression occurs. In turn, subjects with polycythemia vera may benefit from bloodletting [35]. Resolution of AvWS was also observed after the surgical removal of a malignancy, cardiac surgeries (valve replacement) and thyroxin substitution during the course of hypothyroidism [10, 46, 47]. Also withdrawing agents that might contribute to the development of AvWS (ciprofloxacin, griseofulvin, tetracycline, valproic acid, HES) may result in the remission of this condition [45].

Therapeutic agents and procedures used in the management and prevention of bleeding associated with AvWS are listed in Table 4.

Table 4. Therapeutic agents used in the management of AvWS-associated bleeding

\begin{tabular}{|l|l|}
\hline Agent/procedure & Dosage/remarks \\
\hline Desmopressin (Minirin, Ferring) & $\begin{array}{l}0.3 \mu \mathrm{g} / \mathrm{kg} \text { b.w. a(dissolve in } 100 \mathrm{~mL} \text { of } 0.9 \% \mathrm{NaCl} \text { )/intravenous infusion, } 30 \mathrm{~min}, \\
1-2 \text { per day }\end{array}$ \\
\hline vWF concentrates & $30-100$ units/kg b.w. intravenously (frequency depending on vWF activity) \\
\hline rVIIa (NovoSeven, Novo Nordisk) & $90 \mu \mathrm{g} / \mathrm{kg}$ b.w. intravenously every 2-4 h \\
\hline Immunoglobulins (IVIG) & $1 \mathrm{~g} / \mathrm{kg}$ b.w. intravenously for 2 days \\
\hline $\begin{array}{l}\text { Antifibrinolytics - tranexemic acid } \\
\text { (Exacyl, Polfa Warszawa) }\end{array}$ & $20-25 \mathrm{mg} / \mathrm{kg}$ b.w. orally or intravenously every $8-12 \mathrm{~h}$ \\
\hline Plasmapheresis & albumins should be replaced with ffp \\
\hline
\end{tabular}

b.w. - body weight. 
The efficacy of desmopressin (DDAVP) may be limited and short-term, especially in patients who present with autoantibodies against vWF and enhanced clearance of the factor [44]. According to the data from the ISTH registry, the overall proportion of therapeutic responses to DDAVP approximates $32 \%$ and depends on the type of concomitant disease. The worst outcomes were observed in cardiovascular diseases (10\%) and myeloproliferative neoplasms (21\%), whereas autoimmune disorders (33\%), lymphoproliferative neoplasms (44\%) and non-hematological malignancies $(75 \%)$ were shown to be associated with better prognosis $[2,35]$.

Since pharmacokinetics of vWF after administration of desmopressin to individuals with AvWS is difficult to establish, the levels of vWF: Ag and vWF:RCo should be monitored thoroughly during the course of DDAVP therapy [35].

Due to the presence of anti-vWF autoantibodies and enhanced clearance of the factor, plasma-derived vWF concentrates have limited efficacy and exert relatively short therapeutic effect. The half-life of these products is particularly short in individuals with MGUS and in patients with inhibitors [20]. The optimal dose and frequency of a vWF concentrate administration should be based on clinical outcomes, as well as on monitoring vWF activity and concentration [35].

A valuable agent for treatment of hemorrhagic complications observed during the course of AvWS may be recombinant activated factor VII (rVIIa, NovoSeven), already used successfully in the management of hemophiliacs with inhibitor. The efficacy of rVIIa was also confirmed in patients with vWD and alloantibodies against vWF, as well as in subjects with AvWS [48, 49]. Application of rVIIa should be considered especially in subjects resistant to routinely used anti-AvWS therapies [50, 51].

Intravenous immunoglobulins (IVIG) are used primarily in patients in whom AvWS is associated with IgG-MGUS and are generally inefficient in subjects with IgM-MGUS. According to the data from the ISTH registry, patients in whom
AvWS co-exists with lymphoproliferative diseases, autoimmune disorders or neoplasms may also benefit from IVIG therapy [4, 52-54]. Normalization of vWF activity in the plasma is usually observed no earlier than $24-48 \mathrm{~h}$ after the administration of IVIG, and therefore patients with active bleeding and persons qualified to undergo invasive procedures may initially require concomitant administration of other agents (DDAVP, vWF concentrates, rVIIa). If necessary, injection of IVIG can be repeated every 21 days [1].

The aim of plasmapheresis is to eliminate autoantibodies and paraproteins. The procedure is efficient in ca. $20 \%$ of the patients, especially in individuals diagnosed with MGUS. It is particularly recommended in IgM-MGUS, which generally responds poorly to other treatments [55]. To prevent a deficiency of fibrinogen and other coagulation factors, fresh frozen plasma, rather than albumins, should be administered during the course of plasmapheresis. Hemorrhagic complications should be treated with vWF concentrates or desmopressin [35].

Immunosuppressive agents and glucocorticoids are administered to eliminate or block autoantibodies, especially in patients who do did not respond well to other therapies. However, the application of these agents may be limited due to their multiple side effects and delayed therapeutic effect $[14,45]$.

\section{Conclusion}

AvWS is a rare bleeding disorder, usually occurring concomitantly with other conditions. Unfortunately, despite evident clinical manifestation, AvWS is frequently overlooked. Early diagnosis and implementation of an appropriate therapy prevents severe hemorrhagic complications. Aside from control and prevention of hemorrhage, management of AvWS should also focus on the treatment of the primary underlying condition, since achieving its remission is frequently associated with the resolution of the bleeding disorder.

\section{References}

[1] Collins P, Budde U, Rand JH, Federici AB, Kessler CM: Epidemiology and general guidelines of the management of acquired haemophilia and von Willebrand syndrome. Haemophilia 2008, 3, 49-55.

[2] Federici AB: Acquired von Willebrand syndrome: An underdiagnosed and misdiagnosed bleeding complication in patients with lymphoproliferative and myeloproliferative disorders. Semin Hematol 2006, 43, 48-58.

[3] Simone JV, Cornet JA, Abildgaard CF: Acquired von Willebrand's syndrome in systemic lupus erythematosus. Blood 1968, 31, 806-812.

[4] Federici AB, Rand JH, Bucciarelli P, Budde U, van Genderen PJ, Mohri H, Meyer D, Rodeghiero F, Sadler JE: Acquired von Willebrand syndrome: Data from an international registry. Thromb Haemost 2000, 84, 345-349.

[5] Mital A, Prejzner W, Bieniaszewska M, Hellmann A: Prevalence of acquired von Willebrand syndrome during essential thrombocythemia: A retrospective analysis of 170 consecutive patients. Pol Arch Med Wewn 2015, 125, 914-920. 
[6] Mital A, Prejzner W, Hellmann A: Acquired von Willebrand syndrome during the course of myelofibrosis: Analysis of 32 cases. Adv Clin Exp Med 2015, 24, 1001-1006.

[7] Hanratty JM, Cowan CG: Acquired von Willebrand disease secondary to hypothyroidism: A rare cause for postextraction hemorrhage. Oral Surg Oral Med Oral Pathol Oral Radiol Endod 2010, 110, 337-340.

[8] Manfredi E, van Zaane B, Gerdes VE, Brandjes DP, Squizzato A: Hypothyroidism and acquired von Willebrand's syndrome: A systematic review. Haemophilia 2008, 14, 423-433.

[9] Meyer AL, Malehsa D, Bara C, Budde U, Slaughter MS, Haverich A, Strueber M: Acquired von Willebrand syndrome in patients with an axial flow left ventricular assist device. Circ Heart Fail 2010, 3, 675-681.

[10] Vincentelli A, Susen S, Le Tourneau T, Six I, Fabre O, Juthier F, Bauters A, Decoene C, Goudemand J, Prat A, Jude B: Acquired von Willebrand syndrome in aortic stenosis. N Engl J Med 2003, 349, 343-349.

[11] Siaka C, Rugeri L, Caron C, Goudemand J: A new ELISA assay for diagnosis of acquired von Willebrand syndrome. Haemophilia 2003, 9, 303-308.

[12] Bruggers CS, McElligott K, Rallison ML: Acquired von Willebrand disease in twins with autoimmune hypothyroidism: Response to desmopressin and L-thyroxine therapy. J Pediatr 1994, 125, 911-913.

[13] Franchini M, de Gironcoli M, Lippi G, Manzato F, Brazzarola P, Bottura D, Aprili G, Gandini G: Efficacy of desmopressin as surgical prophylaxis in patients with acquired von Willebrand disease undergoing thyroid surgery. Haemophilia 2002, 8, 142-144.

[14] Franchini M, Lippi G: Acquired von Willebrand syndrome: An update. Am J Hematol 2007, 82, 368-375.

[15] Tefferi A, Nichols WL: Acquired von Willebrand disease: Concise review of occurrence, diagnosis, pathogenesis, and treatment. Am J Med 1997, 103, 536-540.

[16] Kumar S, Pruthi RK, Nichols WL: Acquired von Willebrand disease. Mayo Clin Proc 2002, 77, 181-187.

[17] Goudemand J, Samor B, Caron C, Jude B, Gosset D, Mazurier C: Acquired type II von Willebrand's disease: Demonstration of a complexed inhibitor of the von Willebrand factor-platelet interaction and response to treatment. Br J Haematol 1988, 68, 227-233.

[18] Mohri H, Motomura S, Kanamori H, Matsuzaki M, Watanabe S, Maruta A, Kodama F, Okubo T: Clinical significance of inhibitors in acquired von Willebrand syndrome. Blood 1998, 91, 3623-3629.

[19] van Genderen PJ, Vink T, Michiels JJ, van 't Veer MB, Sixma JJ, van Vliet HH: Acquired von Willebrand disease caused by an autoantibody selectively inhibiting the binding of von Willebrand factor to collagen. Blood 1994, 84, $3378-3384$

[20] Federici AB, Stabile F, Castaman G, Canciani MT, Mannucci PM: Treatment of acquired von Willebrand syndrome in patients with monoclonal gammopathy of uncertain significance: Comparison of three different therapeutic approaches. Blood 1998, 92, 2707-2711.

[21] Richard C, Cuadrado MA, Prieto M, Batlle J, Lopez Fernandez MF, Rodriguez Salazar ML, Bello C, Recio M, Santoro T, Gomez Casares MT: Acquired von Willebrand disease in multiple myeloma secondary to absorption of von Willebrand factor by plasma cells. Am J Hematol 1990, 35, 114-117.

[22] Scrobohaci ML, Daniel MT, Levy Y, Marolleau JP, Brouet JC: Expression of GpIb on plasma cells in a patient with monoclonal IgG and acquired von Willebrand disease. Br J Haematol 1993, 84, 471-475.

[23] Budde U, van Genderen PJ: Acquired von Willebrand disease in patients with high platelet counts. Semin Thromb Hemost 1997, 23, 425-431.

[24] O'Brien JR, Etherington MD: Heart valve stenosis and von Willebrand's factor multimers. Lancet 1992, 340, 616.

[25] Strauss RG, Stump DC, Henriksen RA: Hydroxyethyl starch accentuates von Willebrand's disease. Transfusion $1985,25,235-237$.

[26] Gill JC, Wilson AD, Endres-Brooks J, Montgomery RR: Loss of the largest von Willebrand factor multimers from the plasma of patients with congenital cardiac defects. Blood 1986, 67, 758-761.

[27] Pareti FI, Lattuada A, Bressi C, Zanobini M, Sala A, Steffan A, Ruggeri ZM: Proteolysis of von Willebrand factor and shear stress-induced platelet aggregation in patients with aortic valve stenosis. Circulation 2000, 102, $1290-1295$.

[28] Sadler JE: Aortic stenosis, von Willebrand factor, and bleeding. N Engl J Med 2003, 349, 323-325.

[29] van Genderen PJ, Prins FJ, Lucas IS, van de Moesdijk D, van Vliet HH, van Strik R, Michiels JJ: Decreased halflife time of plasma von Willebrand factor collagen binding activity in essential thrombocythaemia: Normalization after cytoreduction of the increased platelet count. Br J Haematol 1997, 99, 832-836.

[30] Castaman G, Lattuada A, Mannucci PM, Rodeghiero F: Characterization of two cases of acquired transitory von Willebrand syndrome with ciprofloxacin: Evidence for heightened proteolysis of von Willebrand factor. Am J Hematol 1995, 49, 83-86.

[31] Castaman G, Rodeghiero F, Lattuada A, La Greca G, Mannucci PM: Multimeric pattern of plasma and platelet von Willebrand factor is normal in uremic patients. Am J Hematol 1993, 44, 266-269.

[32] Federici AB, Berkowitz SD, Lattuada A, Mannucci PM: Degradation of von Willebrand factor in patients with acquired clinical conditions in which there is heightened proteolysis. Blood 1993, 81, 720-725.

[33] Bracey AW, Wu AH, Aceves J, Chow T, Carlile S, Hoots WK: Platelet dysfunction associated with Wilms tumor and hyaluronic acid. Am J Hematol 1987, 24, 247-257.

[34] Michiels J, Schroyens W, Berneman Z, van der Planken M: Atypical variant of acquired von Willebrand syndrome in Wilms tumor: Is hyaluronic acid secreted by nephroblastoma cells the cause? Clin Appl Thromb Hemost 2001, 7, 102-105.

[35] Tiede A, Rand JH, Budde U, Ganser A, Federici AB: How I treat the acquired von Willebrand syndrome. Blood 2011, 117, 6777-6785. 
[36] Mohri H: Acquired von Willebrand syndrome: Its pathophysiology, laboratory features and management. J Thromb Thrombolysis 2003, 15, 141-149.

[37] Favaloro EJ, Facey D, Grispo L: Laboratory assessment of von Willebrand factor. Use of different assays can influence the diagnosis of von Willebrand's disease, dependent on differing sensitivity to sample preparation and differential recognition of high molecular weight VWF forms. Am J Clin Pathol 1995, 104, $264-271$.

[38] Federici AB, Budde U, Rand JH: Acquired von Willebrand syndrome 2004: International Registry - diagnosis and management from online to bedside. Hamostaseologie 2004, 24, 50-55.

[39] Tiede A, Priesack J, Werwitzke S, Bohlmann K, Oortwijn B, Lenting P, Eisert R, Ganser A, Budde U: Diagnostic workup of patients with acquired von Willebrand syndrome: A retrospective single-centre cohort study. J Thromb Haemost 2008, 6, 569-576.

[40] Budde U, Bergmann F, Michiels JJ: Acquired von Willebrand syndrome: experience from 2 years in a single laboratory compared with data from the literature and an international registry. Semin Thromb Hemost 2002, 28, $227-238$.

[41] Budde U, Schneppenheim R, Eikenboom J, Goodeve A, Will K, Drewke E, Castaman G, Rodeghiero F, Federici AB, Batlle J, Perez A, Meyer D, Mazurier C, Goudemand J, Ingerslev J, Habart D, Vorlova Z, Holmberg L, Lethagen S, Pasi J, Hill F, Peake I: Detailed von Willebrand factor multimer analysis in patients with von Willebrand disease in the European study, molecular and clinical markers for the diagnosis and management of type 1 von Willebrand disease (MCMDM-1VWD). J Thromb Haemost 2008, 6, 762-771.

[42] Michiels JJ, Budde U, van der Planken M, van Vliet HH, Schroyens W, Berneman Z: Acquired von Willebrand syndromes: Clinical features, aetiology, pathophysiology, classification and management. Best Pract Res Clin Haematol 2001, 14, 401-436.

[43] Haberichter SL, Castaman G, Budde U, Peake I, Goodeve A, Rodeghiero F, Federici AB, Batlle J, Meyer D, Mazurier C, Goudemand J, Eikenboom J, Schneppenheim R, Ingerslev J, Vorlova Z, Habart D, Holmberg L, Lethagen S, Pasi J, Hill FG, Montgomery RR: Identification of type 1 von Willebrand disease patients with reduced von Willebrand factor survival by assay of the VWF propeptide in the European study: Molecular and clinical markers for the diagnosis and management of type 1 VWD (MCMDM-1VWD). Blood 2008, 111, 4979-4985.

[44] Mannucci PM, Lombardi R, Bader R, Horellou MH, Finazzi G, Besana C, Conard J, Samama M: Studies of the pathophysiology of acquired von Willebrand's disease in seven patients with lymphoproliferative disorders or benign monoclonal gammopathies. Blood 1984, 64, 614-621.

[45] Sucker C, Michiels JJ, Zotz RB: Causes, etiology and diagnosis of acquired von Willebrand disease: A prospective diagnostic workup to establish the most effective therapeutic strategies. Acta Haematol 2009, 121, 177-182.

[46] Dalton RG, Dewar MS, Savidge GF, Kernoff PB, Matthews KB, Greaves M, Preston FE: Hypothyroidism as a cause of acquired von Willebrand's disease. Lancet 1987, 1, 1007-1009.

[47] Scott JP, Montgomery RR, Tubergen DG, Hays T: Acquired von Willebrand's disease in association with Wilm's tumor: Regression following treatment. Blood 1981, 58, 665-669.

[48] Franchini M, Veneri D, Lippi G: The use of recombinant activated factor VII in congenital and acquired von Willebrand disease. Blood Coagul Fibrinolysis 2006, 17, 615-619.

[49] Sucker C, Scharf RE, Zotz RB: Use of recombinant factor VIIa in inherited and acquired von Willebrand disease. Clin Appl Thromb Hemost 2009, 15, 27-31.

[50] Friederich PW, Wever PC, Briet E, Doorenbos CJ, Levi M: Successful treatment with recombinant factor VIIa of therapy-resistant severe bleeding in a patient with acquired von Willebrand disease. Am J Hematol 2001, 66, $292-294$.

[51] Smaradottir A, Bona R: A case of acquired von Willebrand syndrome successfully treated with recombinant Factor VIIa during thyroidectomy. Thromb Haemost 2004, 92, 666-667.

[52] Delannoy A, Saillez AC: High-dose intravenous gammaglobulin for acquired von Willebrand's disease. $\mathrm{Br}$ J Haematol 1988, 70, 387.

[53] Federici AB: Use of intravenous immunoglobulin in patients with acquired von Willebrand syndrome. Hum Immunol 2005, 66, 422-430.

[54] Michiels JJ, van Vliet HH: Acquired von Willebrand disease in monoclonal gammapathies: Effectiveness of highdose intravenous gamma globulin. Clin Appl Thromb Hemost 1999, 5, 152-157.

[55] Federici AB: Therapeutic approaches to acquired von Willebrand syndrome. Expert Opin Investig Drugs 2000, 9, 347-354.

\section{Address for correspondence:}

Andrzej Mital

Department of Hematology and Transplantology

Medical University of Gdańsk

ul. Dębinki 7

80-952 Gdańsk

Poland

Tel.: +48 583492230

E-mail: amital@wp.pl

Received: 14.02 .2016

Conflict of interest: None declared

Revised: 8.05.2016

Accepted: 1.09.2016 